\title{
Metathesis and Metallotropy: A Versatile Combination for the Synthesis of Oligoenynes
}

\author{
Mansuk Kim and Daesung Lee* \\ Department of Chemistry, University of Wisconsin, Madison, WI 53706 \\ dlee@chem.wisc.edu
}

Supporting Information

\section{Materials and Methods}

${ }^{1} \mathrm{H}$ and ${ }^{13} \mathrm{C}$ NMR spectra were recorded on a Bruker $\mathrm{AC}-250$ or a Bruker AC-300 spectrometer. Tetramethylsilane (TMS) served as an internal standard $(\delta=0)$ for ${ }^{1} \mathrm{H}$ NMR and $\mathrm{CDCl}_{3}$ was used as an internal standard $(\delta=77.23)$ for ${ }^{13} \mathrm{C}$ NMR. All reactions were run under an atmosphere of nitrogen, unless otherwise indicated. Flasks were oven-dried overnight and cooled under a stream of nitrogen. Compounds were purchased from Aldrich. Dichloromethane was distilled from $\mathrm{CaH}_{2}$. All other solvents were purified based on standard procedures. Flash chromatography was performed using silica gel $60 \AA$ (32-63 mesh) from Sorbent Technologies. Reactions were monitored by thin layer chromatography (TLC) using $0.25 \mathrm{~mm}$ E. Merck precoated silica gel 60 (particle size $0.040-0.063 \mathrm{~mm}$ ). 


\section{General scheme for the preparation of the substrates for}

Metathesis and Metallotropy (M\&M) - Note: Yields were not optimized extensively.

- $\mathrm{Cu}(\mathrm{I})$-mediated coupling reaction was performed according to the literature procedures. ${ }^{1}$

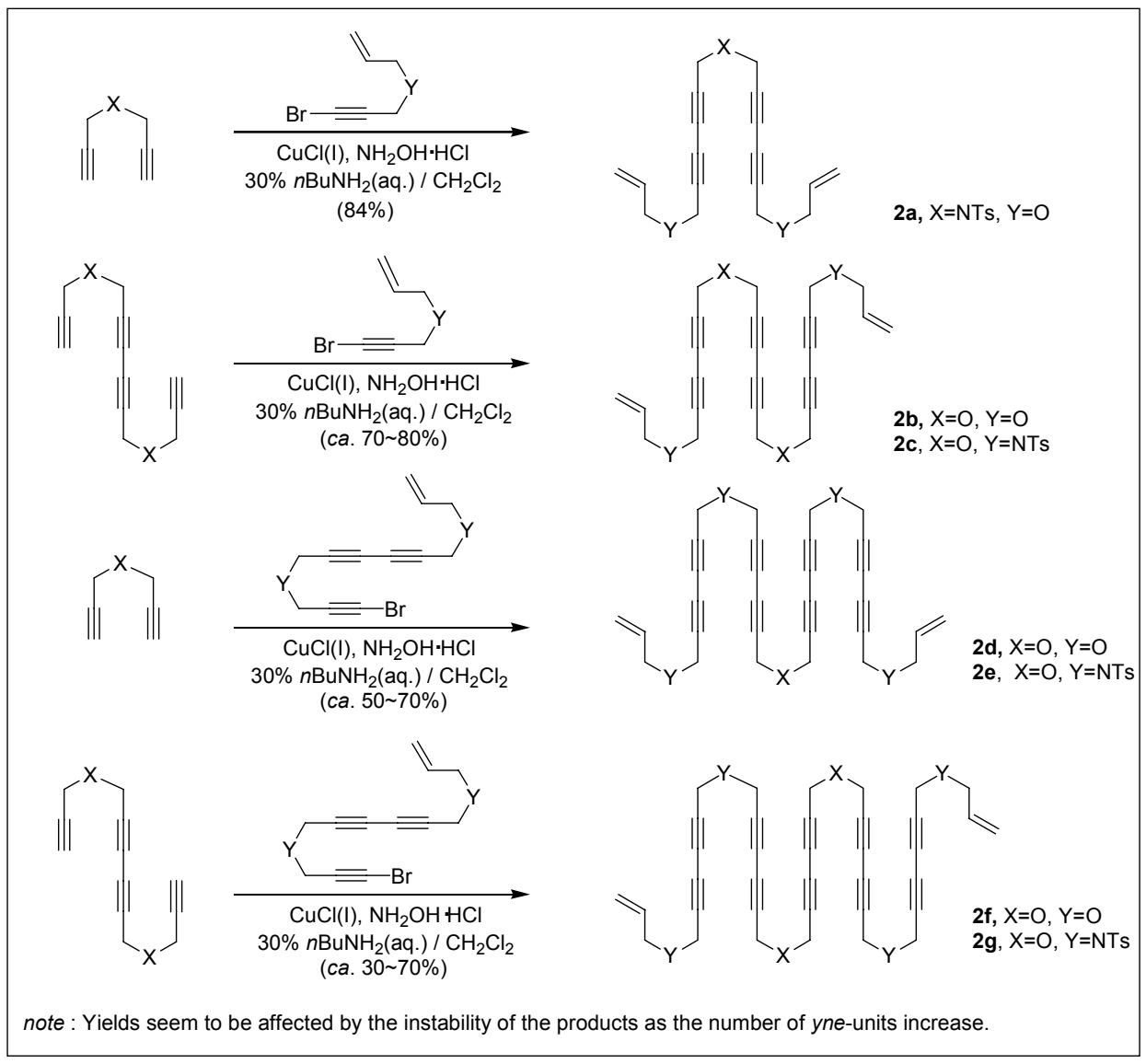

\section{Representative procedure for $\mathrm{Cu}(\mathrm{I})$-mediated coupling reaction}

To a stirred solution of $n-\mathrm{BuNH}_{2}(0.9 \mathrm{ml})$ and distilled water $(2.1 \mathrm{ml})$ was added copper(I) chloride ( 0.2 equiv) at $0{ }^{\circ} \mathrm{C}$, which resulted in a deep blue solution. A few crystals of $\mathrm{NH}_{2} \mathrm{OH} \cdot \mathrm{HCl}$ were added to get a colorless solution which is indicative of the presence of the required $\mathrm{Cu}(\mathrm{I})$ salt. At the same temperature was added 4-methyl- $N, N$-diprop-2-ynylbenzenesulfonamide ( $257 \mathrm{mg}, 0.099 \mathrm{mmol}$ ) by a syringe as a solution in $\mathrm{CH}_{2} \mathrm{Cl}_{2}$ upon which was a yellow suspension formed. Then a freshly prepared 3-(3-bromoprop-2-ynyloxy)-propene (2.5 equiv) was slowly added under a flow of $\mathrm{N}_{2}$ in order to prevent the solution from turning to green

1 (a) Marino, J. P.; Nguyen, H. N. J. Org. Chem. 2002, 67, 6841. (b) Kim, S.; Kim, S.; Lee, T.; Ko, H.; Kim, D. Org. Lett. 2004, 6, 3601, and references cited therein. 
or blue (Tip: $\mathrm{NH}_{2} \mathrm{OH} \cdot \mathrm{HCl}$ can be added as necessary throughout the reaction). The reaction mixture was allowed to warm up to room temperature. After stirring for 10 20 minutes, the brownish orange colored solution was extracted twice with $\mathrm{CH}_{2} \mathrm{Cl}_{2}$, dried over magnesium sulfate, and concentrated in vacuo. The crude product was purified by flash column chromatography using pentane/ethyl acetate (gradient elusion) to yield the desired product $\mathbf{2 a}$ (red oil, $45 \mathrm{mg}, 84 \%$ based on terminal diyne). - Note: Even with extreme caution, homocoupling of the bromoalkyne to generate the undesired 1,6-bis-allyloxyhexa-2,4-diyne could not be excluded.

\section{Representative procedure for M\&M}

Diene-1,3-diyne 2a (87 mg, $0.099 \mathrm{mmol})$ was dissolved in freshly distilled $\mathrm{CH}_{2} \mathrm{Cl}_{2}(0.002 \mathrm{M})$ and Grubbs $2^{\text {nd }}$ generation catalyst 1 , $\left(\mathrm{H}_{2} \mathrm{Imes}\right)\left(\mathrm{PCy}_{3}\right) \mathrm{Cl}_{2} \mathrm{Ru}=\mathrm{CHPh}(4.2 \mathrm{mg}, 0.005 \mathrm{mmol}, 5$ mol \%) was added. After refluxing under nitrogen for 4 hours, the reaction mixture was concentrated under reduced pressure. The residue was purified by flash column chromatography using pentane/ether $/ \mathrm{CH}_{2} \mathrm{Cl}_{2}$ (gradient elusion) to yield the desired M\&M product 3a (solid, 45 $\mathrm{mg}, 88 \%$ ). - Note: In order to get a decent level of solubility during elution, it is recommended to use $\mathrm{CH}_{2} \mathrm{Cl}_{2}$ for the eluent.
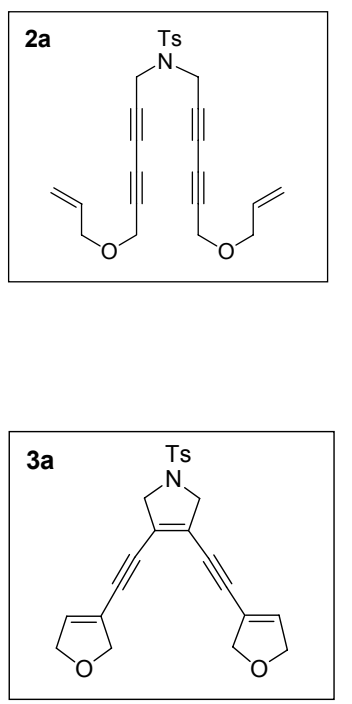

${ }^{1}$ H NMR (300 MHz, CDCl $) \delta 7.69(\mathrm{~d}, J=8.4 \mathrm{~Hz}, 2 \mathrm{H}), 7.32(\mathrm{~d}, J=8.4$ $\mathrm{Hz}, 2 \mathrm{H}), 5.87$ (ddt, $J=17.2,10.4,5.8 \mathrm{~Hz}, 2 \mathrm{H}), 5.31(\mathrm{ddd}, J=17.2,1.5 \mathrm{~Hz}$, 2H), 5.23 (ddd, J = 10.4, $1.5 \mathrm{~Hz}, 2 \mathrm{H}), 4.21(\mathrm{~s}, 4 \mathrm{H}), 4.18$ (s, 4H), 4.03 (ddd, $J=5.8,1.5 \mathrm{~Hz}, 4 \mathrm{H}), 2.42(\mathrm{~s}, 3 \mathrm{H}) ;{ }^{13} \mathbf{C}$ NMR (75 MHz, $\left.\mathbf{C D C l}_{3}\right) \delta 144.57$, $134.72,133.72,129.21,127.95,118.37,75.40,71.64,70.99,70.26,70.12$, 57.58, 37.48, 21.72; MS (FAB, $m / z$ ) calcd for $\mathrm{C}_{25} \mathrm{H}_{25} \mathrm{NO}_{4} \mathrm{~S}[\mathrm{M}+\mathrm{H}]^{+} 436$, found 436 .

${ }^{1}$ H NMR (300 MHz, $\left.\mathbf{C D C l}_{3}\right) \delta 7.72(\mathrm{~d}, J=8.5 \mathrm{~Hz}, 2 \mathrm{H}), 7.34(\mathrm{~d}, J=8.5$ $\mathrm{Hz}, 2 \mathrm{H}), 6.22$ (tt, $J=2.4 \mathrm{~Hz}, 2 \mathrm{H}), 4.78$ (dd, $J=4.9,2.4 \mathrm{~Hz}, 1 \mathrm{H}), 4.76$ (dd, $J=4.9,2.4 \mathrm{~Hz}, 1 \mathrm{H}), 4.62(\mathrm{dd}, J=5.0,2.4 \mathrm{~Hz}, 1 \mathrm{H}), 4.60(\mathrm{dd}, J=5.0,2.4$ $\mathrm{Hz}, 1 \mathrm{H}), 4.21(\mathrm{~s}, 4 \mathrm{H}), 2.44(\mathrm{~s}, 3 \mathrm{H}) ;{ }^{13} \mathbf{C}$ NMR (63 MHz, CDCl $\left.\mathbf{3}\right) \delta 144.27$, $134.26,133.61,130.18,127.71,124.31,120.77,91.00,85.66,76.72$, 76.57, 56.68, 21.74; MS (FAB, $m / z)$ calcd for $\mathrm{C}_{23} \mathrm{H}_{21} \mathrm{NO}_{4} \mathrm{~S}[\mathrm{M}+\mathrm{H}]^{+} 408$, found 408 .

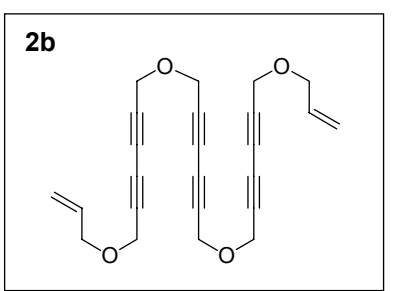

${ }^{1}$ H NMR (300 MHz, $\mathbf{C D C l}_{3}$ ) $\delta 5.90$ (ddt, $\left.J=17.1,10.2,5.6 \mathrm{~Hz}, 2 \mathrm{H}\right)$, $5.33(\mathrm{ddt}, J=17.1,1.7 \mathrm{~Hz}, 2 \mathrm{H}), 5.25(\mathrm{ddt}, J=10.2,1.7 \mathrm{~Hz}, 2 \mathrm{H}), 4.35$ (s, 8H), $4.24(\mathrm{~s}, 4 \mathrm{H}), 4.07(\mathrm{dt}, J=5.6,1.7 \mathrm{~Hz}, 4 \mathrm{H}) ;{ }^{13} \mathbf{C}$ NMR (75 $\left.\mathbf{M H z}, \mathbf{C D C l}_{3}\right) \delta 133.83,118.44,76.10,74.78,74.22,71.50,71.25$, 71.05, 70.38, 57.73, 57.39, 57.30; MS (FAB, $m / z)$ calcd for $\mathrm{C}_{24} \mathrm{H}_{22} \mathrm{O}_{4}$ $[\mathrm{M}+\mathrm{H}]^{+} 375 /[\mathrm{M}+\mathrm{Na}] 397$, found $375 / 397$, respectively. 

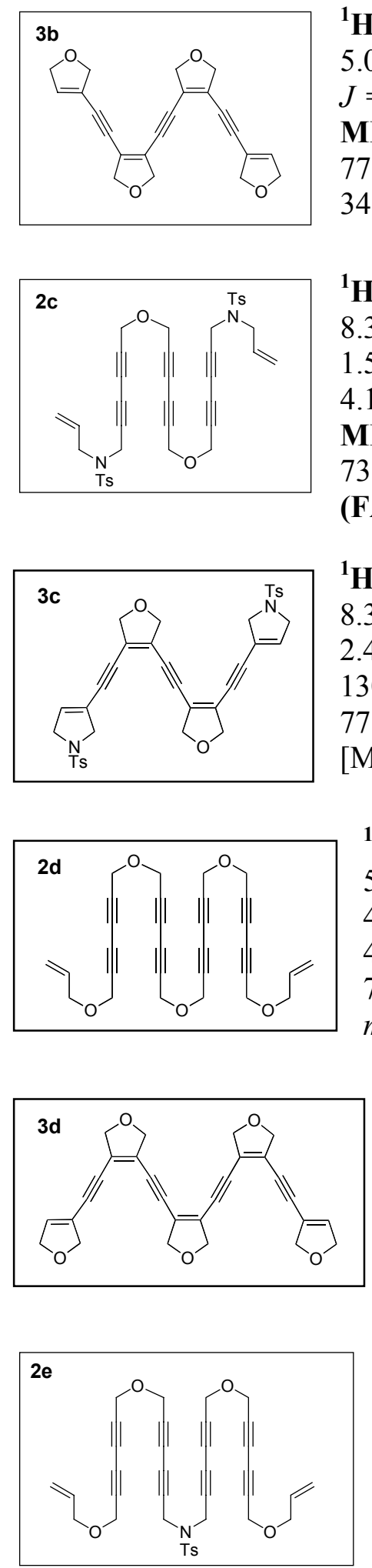

${ }^{1}$ H NMR (300 MHz, $\left.\mathbf{C D C l}_{3}\right) \delta 6.24(\mathrm{tt}, J=2.1 \mathrm{~Hz}, 2 \mathrm{H}), 4.79(\mathrm{dd}, J=$ $5.0,2.1 \mathrm{~Hz}, 2 \mathrm{H}), 4.77(\mathrm{dd}, J=5.0,2.1 \mathrm{~Hz}, 2 \mathrm{H}), 4.75(\mathrm{~s}, 8 \mathrm{H}), 4.66(\mathrm{dd}$, $J=5.0,2.1 \mathrm{~Hz}, 2 \mathrm{H}), 4.64(\mathrm{dd}, J=5.0,2.4 \mathrm{~Hz}, 2 \mathrm{H}) ;{ }^{13} \mathbf{C}$ NMR $(75$ MHz, $\left.\mathbf{C D C l}_{3}\right) \delta 134.02,127.08,125.87,121.13,92.04,90.78,85.21$, 77.64, 77.47, 76.86, 76.62; MS (DEI, $m / z$ ) calcd for $\mathrm{C}_{22} \mathrm{H}_{18} \mathrm{O}_{4}[\mathrm{M}]^{+}$ 346 , found 346 .

${ }^{1} \mathbf{H}$ NMR (300 MHz, CDCl $) \delta 7.73(\mathrm{~d}, J=8.3 \mathrm{~Hz}, 4 \mathrm{H}), 7.34(\mathrm{~d}, J=$ $8.3 \mathrm{~Hz}, 4 \mathrm{H}$ ), 5.73 (ddt, $\mathrm{J}=17.2,9.9,6.6 \mathrm{~Hz}, 2 \mathrm{H}$ ), 5.30 (ddt, $J=17.2$, $1.5 \mathrm{~Hz}, 2 \mathrm{H}), 5.26$ (ddt, $J=9.9,1.5 \mathrm{~Hz}, 2 \mathrm{H}), 4.31(\mathrm{~s}, 4 \mathrm{H}), 4.28(\mathrm{~s}, 4 \mathrm{H})$, $4.16(\mathrm{~s}, 4 \mathrm{H}), 3.80(\mathrm{~d}, J=6.6 \mathrm{~Hz}, 4 \mathrm{H}), 2.45(\mathrm{~s}, 6 \mathrm{H}) ;{ }^{13} \mathbf{C}$ NMR (75 MHz, $\left.\mathbf{C D C l}_{3}\right) \delta 144.04,135.73,131.87,129.80,127.87,120.46,74.75$, 73.46, 73.03, 71.20, 71.14, 69.51, 57.30, 49.64, 36.67, 21.72; MS (FAB, $m / z$ ) calcd for $\mathrm{C}_{38} \mathrm{H}_{36} \mathrm{~N}_{2} \mathrm{O}_{6} \mathrm{~S}_{2}[\mathrm{M}+\mathrm{H}]^{+} 681$, found 681 .

${ }^{1} \mathbf{H}$ NMR (300 MHz, CDCl $\left.{ }_{3}\right) \delta 7.73(\mathrm{~d}, J=8.3 \mathrm{~Hz}, 4 \mathrm{H}), 7.35(\mathrm{~d}, J=$ $8.3 \mathrm{~Hz}, 4 \mathrm{H}), 5.98(\mathrm{~s}, 2 \mathrm{H}), 4.73(\mathrm{~s}, 8 \mathrm{H}), 4.22(\mathrm{~m}, 4 \mathrm{H}), 4.14(\mathrm{~m}, 4 \mathrm{H})$, $2.45(\mathrm{~s}, 6 \mathrm{H}) ;{ }^{13} \mathbf{C}$ NMR (75 MHz, $\left.\mathbf{C D C l}_{3}\right) \delta 144.10,134.04,132.58$, $130.14,127.70,126.81,126.45,119.96,92.21,90.77,84.73,77.50$, 77.43, 56.54, 55.48, 21.76; MS (FAB, $m / z$ ) calcd for $\mathrm{C}_{36} \mathrm{H}_{32} \mathrm{~N}_{2} \mathrm{O}_{6} \mathrm{~S}_{2}$ $[\mathrm{M}+\mathrm{H}]^{+} 653$, found 653 .

${ }^{1}$ H NMR (300 MHz, $\left.\mathbf{C D C l}_{3}\right) \delta 5.89(\mathrm{ddt}, J=17.2,10.5,5.9 \mathrm{~Hz}, 2 \mathrm{H})$, $5.32(\mathrm{ddd}, J=17.2,1.6 \mathrm{~Hz}, 2 \mathrm{H}), 5.24$ (ddd, $J=10.5,1.6 \mathrm{~Hz}, 2 \mathrm{H}$ ), $4.34(\mathrm{~s}, 8 \mathrm{H}), 4.34(\mathrm{~s}, 4 \mathrm{H}), 4.23(\mathrm{~s}, 4 \mathrm{H}), 4.07$ (ddd, $J=5.9,1.6 \mathrm{~Hz}$, 4H); ${ }^{13} \mathbf{C}$ NMR (75 MHz, $\left.\mathbf{C D C l}_{3}\right) \delta 133.8,118.5,76.1,74.8,74.7$, 74.2, 71.5, 71.3, 71.2, 71.1, 70.4, 57.7, 57.4, 57.4, 57.3; MS (FAB, $m / z$ ) calcd for $\mathrm{C}_{30} \mathrm{H}_{26} \mathrm{O}_{5}[\mathrm{M}+\mathrm{Na}]^{+} 489$, found 489 .

${ }^{1}$ H NMR (300 MHz, $\left.\mathbf{C D C l}_{3}\right) \delta 6.24(\mathrm{tt}, J=2.1 \mathrm{~Hz}, 2 \mathrm{H}), 4.78(\mathrm{~s}$, $4 \mathrm{H}) 4.78(\mathrm{dd}, J=5.1,2.1 \mathrm{~Hz}, 2 \mathrm{H}), 4.77(\mathrm{dd}, J=5.1,2.1 \mathrm{~Hz}, 2 \mathrm{H})$, $4.74(\mathrm{~s}, 8 \mathrm{H}), 4.66(\mathrm{dd}, J=5.1,2.1 \mathrm{~Hz}, 2 \mathrm{H}), 4.65(\mathrm{dd}, J=5.1,2.4$ $\mathrm{Hz}, 2 \mathrm{H}) ;{ }^{13} \mathbf{C}$ NMR (75 MHz, $\left.\mathbf{C D C l}_{3}\right) \delta 134.2,127.4,126.8,125.8$, 121.1, 92.3, 91.5, 90.8, 85.2, 77.7, 77.5, 76.9, 76.7; HRMS (FAB, $m / z$ ) calcd for $\mathrm{C}_{28} \mathrm{H}_{22} \mathrm{O}_{5}[\mathrm{M}+\mathrm{H}]^{+} 439.1545$, found 439.1537 .

${ }^{1}$ H NMR (300 MHz, $\left.\mathbf{C D C l}_{3}\right) \delta 7,71(\mathrm{~d}, J=8.3 \mathrm{~Hz}, 2 \mathrm{H}), 7.35(\mathrm{~d}, J$ $=8.3 \mathrm{~Hz}, 2 \mathrm{H}), 5.90(\mathrm{ddt}, J=17.2,10.4,6.0 \mathrm{~Hz}, 2 \mathrm{H}), 5.33$ (ddt, J = $17.2,1.5 \mathrm{~Hz}, 2 \mathrm{H}), 5.15$ (ddt, $J=10.4,1.5 \mathrm{~Hz}, 2 \mathrm{H}), 4.32$ (s, 4H), $4.30(\mathrm{~s}, 4 \mathrm{H}), 4.25(\mathrm{~s}, 4 \mathrm{H}), 4.22(\mathrm{~s}, 4 \mathrm{H}), 4.07(\mathrm{dt}, J=6.0,1.5 \mathrm{~Hz}$, $4 \mathrm{H}), 2.46(\mathrm{~s}, 3 \mathrm{H})$. - Note : compound $2 \mathrm{e}$ is very unstable forming a 
red-colored film, so that it was used directly for the next transformation without full characterization.

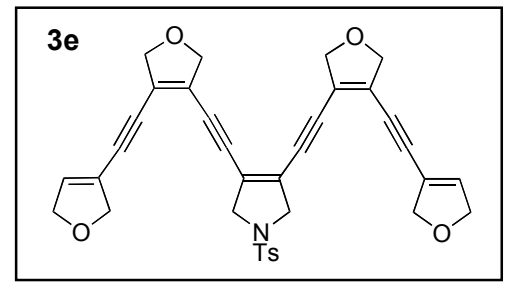

${ }^{1} \mathbf{H}$ NMR (300 MHz, $\left.\mathbf{C D C l}_{3}\right) \delta 7.73(\mathrm{~d}, J=8.3 \mathrm{~Hz}, 2 \mathrm{H}), 7.36(\mathrm{~d}$, $J=8.3 \mathrm{~Hz}, 2 \mathrm{H}), 6.24(\mathrm{tt}, J=1.9 \mathrm{~Hz}, 2 \mathrm{H}), 4.79(\mathrm{dd}, J=5.1,2.1$ $\mathrm{Hz}, 2 \mathrm{H}), 4.78(\mathrm{dd}, J=5.1,2.1 \mathrm{~Hz}, 2 \mathrm{H}), 4.71(\mathrm{~s}, 8 \mathrm{H}), 4.63(\mathrm{dd}, J$ $=5.1,2.1 \mathrm{~Hz}, 2 \mathrm{H}), 4.61(\mathrm{dd}, J=5.1,2.1 \mathrm{~Hz}, 2 \mathrm{H}), 4.26(\mathrm{~s}, 4 \mathrm{H})$, $2.45(\mathrm{~s}, 3 \mathrm{H}) ;{ }^{13} \mathbf{C}$ NMR (75 MHz, CDCl 3 ) $\delta$ 144.4, 134.4, 133.7, $130.3,127.99,127.76,125.27,124.9,121.0,92.4,91.2,90.9$, 85.0, 77.4, 76.6, 56.8, 21.8; MS (MALDI, matrix = 2,5-dihydroxybenzoic acid, $\mathrm{m} / \mathrm{z}$ ) calcd for $\mathrm{C}_{35} \mathrm{H}_{29} \mathrm{NO}_{6} \mathrm{~S}[\mathrm{M}+\mathrm{H}]^{+}$592.2, found 592.1

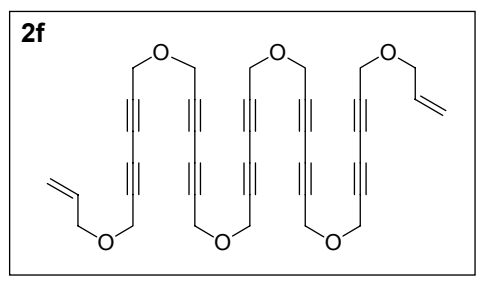

${ }^{1} \mathbf{H}$ NMR (300 MHz, $\left.\mathbf{C D C l}_{3}\right) \delta 5.88(\mathrm{ddt}, J=17.2,10.4,5.7 \mathrm{~Hz}$, $2 \mathrm{H}$ ), 5.32 (ddt, $J=17.2,1.6 \mathrm{~Hz}, 2 \mathrm{H}$ ), 5.23 (ddt, $J=10.4,1.6 \mathrm{~Hz}$, $2 \mathrm{H}), 4.33(\mathrm{~s}, 16 \mathrm{H}), 4.23(\mathrm{~s}, 4 \mathrm{H}), 4.06(\mathrm{dt}, J=5.7,1.6 \mathrm{~Hz}, 4 \mathrm{H})$; ${ }^{13}$ C NMR (75 MHz, $\left.\mathbf{C D C l}_{3}\right): \delta 133.8,118.4,76.1,74.8,74.7$, 74.7, 74.2, 71.5, 71.3, 71.2, 71.0, 70.4, 57.7, 57.4, 57.4, 57.3; MS (FAB, $m / z$ ) calcd for $\mathrm{C}_{36} \mathrm{H}_{30} \mathrm{O}_{6}[\mathrm{M}+\mathrm{Na}]^{+}$581, found 581 .

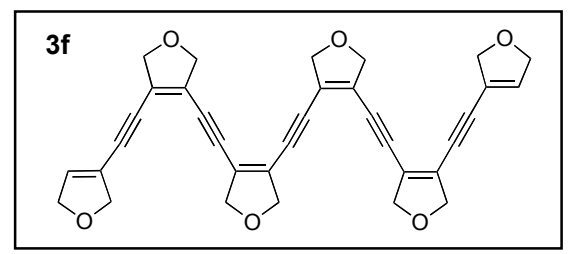

${ }^{1} \mathbf{H}$ NMR (300 MHz, $\left.\mathbf{C D C l}_{3}\right) \delta 6.24(\mathrm{tt}, J=2.1 \mathrm{~Hz}, 2 \mathrm{H})$, $4.78(\mathrm{dd}, J=5.1,2.1 \mathrm{~Hz}, 2 \mathrm{H}), 4.77(\mathrm{dd}, J=5.1,2.1 \mathrm{~Hz}, 2 \mathrm{H})$, $4.76(\mathrm{~s}, 8 \mathrm{H}), 4.74(\mathrm{~s}, 8 \mathrm{H}) 4.65(\mathrm{dd}, J=5.1,2.1 \mathrm{~Hz}, 2 \mathrm{H}), 4.64$ $(\mathrm{dd}, J=5.1,2.1 \mathrm{~Hz}, 2 \mathrm{H}) ;{ }^{13} \mathbf{C}$ NMR (75 MHz, $\left.\mathbf{C D C l}_{3}\right) \delta$ $134.2,127.5,127.2,126.7,125.9,121.2,92.3,91.6,91.4$, 90.8, 85.3, 77.5, 77.4, 76.7; MS (MALDI, $\mathrm{m} / \mathrm{z}$ ) calcd for $\left.\mathrm{C}_{34} \mathrm{H}_{26} \mathrm{O}_{6}\left[\mathrm{M}+{ }^{107} \mathrm{Ag}\right] /\left[\mathrm{M}+{ }^{109} \mathrm{Ag}\right]\right]^{+} 637.1 / 639.5$, found $637.7 / 639.7$.

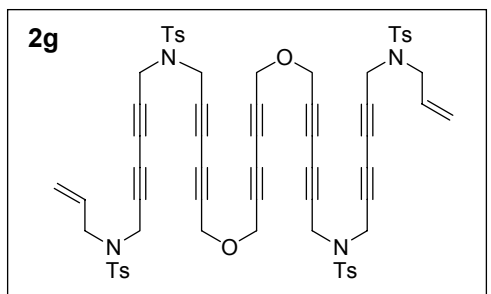

${ }^{1}$ H NMR (300 MHz, $\left.\mathbf{C D C l}_{3}\right) \delta 7.69(\mathrm{~d}, J=8.3 \mathrm{~Hz}, 4 \mathrm{H}), 7.66(\mathrm{~d}$, $J=8.3 \mathrm{~Hz}, 4 \mathrm{H}), 7.31(2 \times \mathrm{d}, J=8.3,8 \mathrm{H}), 5.68(\mathrm{ddt}, J=16.9$, $10.9,6.5 \mathrm{~Hz}, 2 \mathrm{H}), 5.28(\mathrm{~d}, J=16.9 \mathrm{~Hz}, 2 \mathrm{H}), 5.27(\mathrm{~d}, J=10.9 \mathrm{~Hz}$, 2H), 4.31 (s, 4H), 4.29 (s, 4H), $4.19(\mathrm{~s}, 4 \mathrm{H}), 4.15$ (s, 4H), 4.12 (s, $4 \mathrm{H}), 3.76(\mathrm{~d}, J=6.5 \mathrm{~Hz}, 4 \mathrm{H}), 2.45(\mathrm{~s}, 6 \mathrm{H}), 2.44(\mathrm{~s}, 6 \mathrm{H}) ;{ }^{13} \mathbf{C}$ NMR (75 MHz, $\left.\mathbf{C D C l}_{3}\right) \delta 144.7,144.1,135.8,134.8,131.9$, $130.0,129.8,128.0,127.9,120.5,74.7,74.2,72.6,71.3,71.2$, 71.0, 70.1, 70.0, 69.4, 57.4, 57.3, 49.7, 37.5, 37.4, 36,6, 21.8

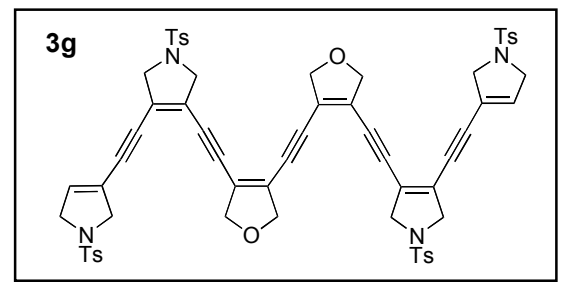

${ }^{1}$ H NMR (300 MHz, CDCl $\left.\mathbf{l}_{3}\right) \delta .69(\mathrm{~d}, J=8.3 \mathrm{~Hz}, 8 \mathrm{H}), 7.32$ $(\mathrm{d}, J=8.3 \mathrm{~Hz}, 8 \mathrm{H}), 5.96(\mathrm{~s}, 2 \mathrm{H}), 4.72(\mathrm{~s}, 8 \mathrm{H}), 4.18(\mathrm{~s}, 8 \mathrm{H})$, 
$4.17(\mathrm{~m}, 4 \mathrm{H}), 4.10(\mathrm{~m}, 4 \mathrm{H}), 2.43(\mathrm{~s}, 12 \mathrm{H}) ;{ }^{13} \mathbf{C}$ NMR (75 MHz, $\left.\mathbf{C D C l}_{3}\right) \delta$ 144.3, 144.1, 134.0, 133.7, 133.3, 130.2, 130.1, 127.8, 127.7, 127.4, 126.8, 125.1, 124.6, 119.8, 91.9, 91.5, 90.5, 85.3, 77.4, 56.7, 56.4, 55.5, 21.8; MS (FAB, $m / z$ ) calcd for $\mathrm{C}_{62} \mathrm{H}_{54} \mathrm{~N}_{4} \mathrm{O}_{5} \mathrm{~S}_{4}[\mathrm{M}+\mathrm{H}]^{+} 1143$, found 1143 .

\section{Scheme for the synthesis of $4 a$ and $4 b$}
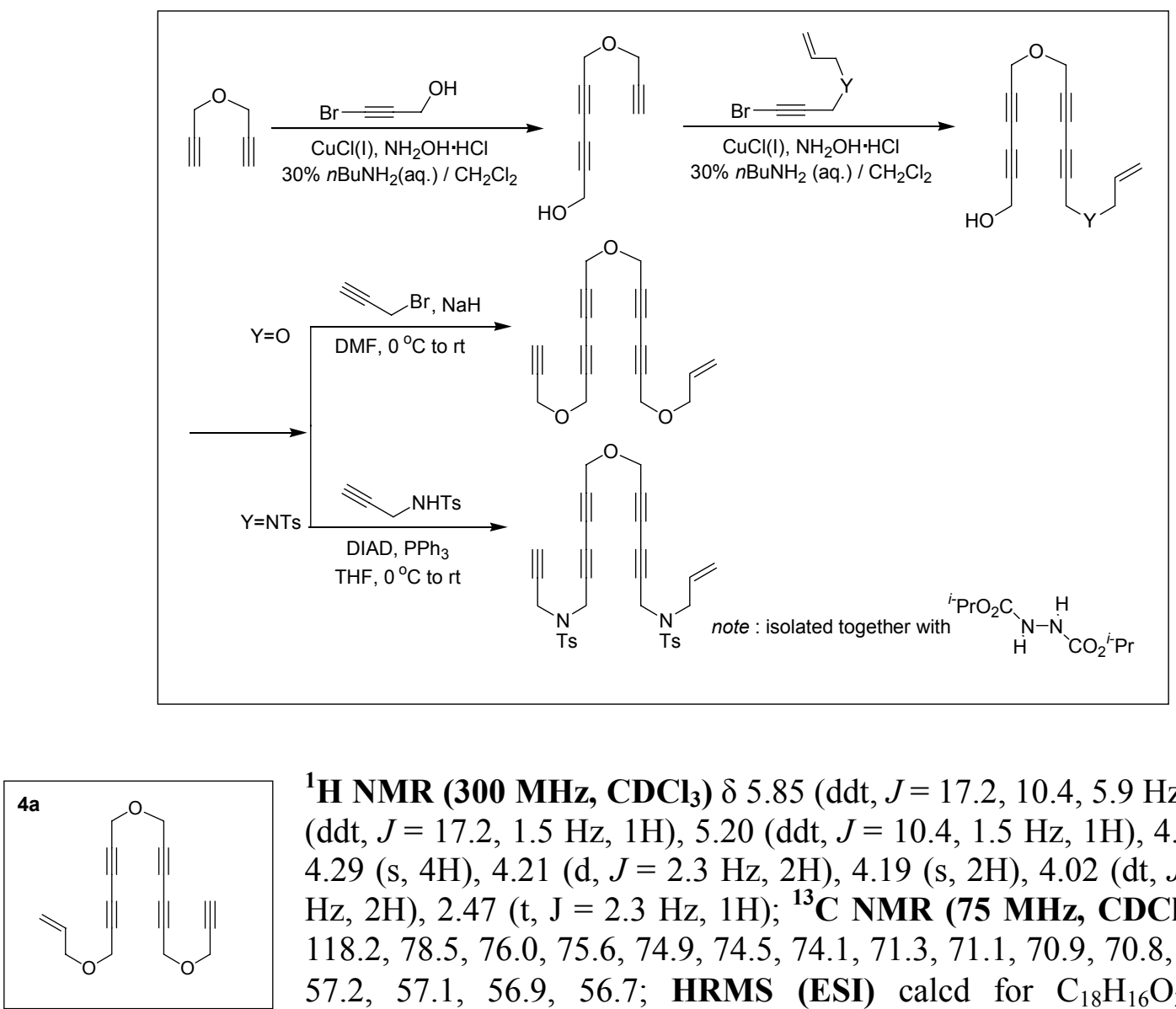

${ }^{1}$ H NMR (300 MHz, CDCl 3 ) $\delta 5.85$ (ddt, $\left.J=17.2,10.4,5.9 \mathrm{~Hz}, 1 \mathrm{H}\right), 5.28$ (ddt, $J=17.2,1.5 \mathrm{~Hz}, 1 \mathrm{H}), 5.20(\mathrm{ddt}, J=10.4,1.5 \mathrm{~Hz}, 1 \mathrm{H}), 4.30(\mathrm{~s}, 2 \mathrm{H})$, $4.29(\mathrm{~s}, 4 \mathrm{H}), 4.21(\mathrm{~d}, J=2.3 \mathrm{~Hz}, 2 \mathrm{H}), 4.19(\mathrm{~s}, 2 \mathrm{H}), 4.02(\mathrm{dt}, J=5.9,1.5$ $\mathrm{Hz}, 2 \mathrm{H}), 2.47(\mathrm{t}, \mathrm{J}=2.3 \mathrm{~Hz}, 1 \mathrm{H}) ;{ }^{13} \mathbf{C}$ NMR (75 MHz, $\left.\mathbf{C D C l}_{3}\right) \delta 133.7$, $118.2,78.5,76.0,75.6,74.9,74.5,74.1,71.3,71.1,70.9,70.8,70.2,57.5$, 57.2, 57.1, 56.9, 56.7; HRMS (ESI) calcd for $\mathrm{C}_{18} \mathrm{H}_{16} \mathrm{O}_{3}[\mathrm{M}+\mathrm{H}]^{\bullet+}$ 281.1178 , found 281.1169 .

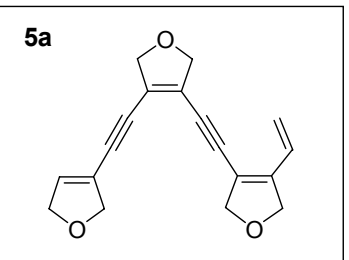

${ }^{1}$ H NMR (300 MHz, CDCl $) \delta 6.76(\mathrm{dd}, J=17.8,11.5 \mathrm{~Hz}, 1 \mathrm{H}), 6.24(\mathrm{tt}$, $J=2.3 \mathrm{~Hz}, 1 \mathrm{H}), 5.34(\mathrm{~d}, J=11.5 \mathrm{~Hz}, 1 \mathrm{H}), 5.18(\mathrm{~d}, J=17.8 \mathrm{~Hz}, 1 \mathrm{H})$, $4.90(\mathrm{~d}, J=3.8 \mathrm{~Hz}, 1 \mathrm{H}), 4.88(\mathrm{~d}, J=3.8 \mathrm{~Hz}, 1 \mathrm{H}), 4.80(\mathrm{dd}, J=3.8,2.3$ $\mathrm{Hz}, 1 \mathrm{H}), 4.79(\mathrm{dd}, J=3.8,2.3 \mathrm{~Hz}, 1 \mathrm{H}), 4.76(\mathrm{~s}, 4 \mathrm{H}), 4.74(\mathrm{~d}, J=3.8 \mathrm{~Hz}$, $1 \mathrm{H}), 4.73$ (d, $J=3.8 \mathrm{~Hz}, 1 \mathrm{H}), 4.68$ (dd, $J=3.8,2.3 \mathrm{~Hz}, 1 \mathrm{H}), 4.66$ (dd, $J$ found 281.2383 . $=3.8,2.3 \mathrm{~Hz}, 1 \mathrm{H})$; HRMS (ESI) calcd for $\mathrm{C}_{18} \mathrm{H}_{16} \mathrm{O}_{3}[\mathrm{M}+\mathrm{H}]^{\bullet+}$ 281.2481, 


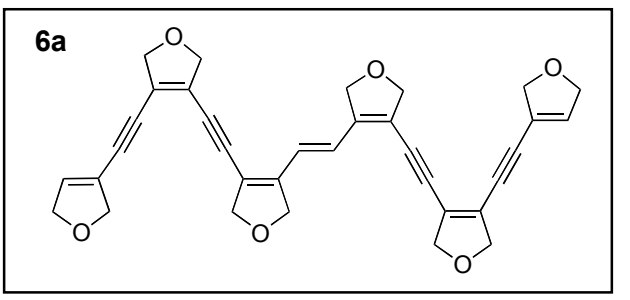

$85.2,77.4,76.9,76.7,75.2$
${ }^{1}$ H NMR (300 MHz, CDCl $\left.)_{3}\right) 6.45(\mathrm{~s}, 2 \mathrm{H}), 6.24(\mathrm{tt}, J=$ $2.2 \mathrm{~Hz}, 2 \mathrm{H}), 4.90(\mathrm{~d}, J=3.8 \mathrm{~Hz}, 2 \mathrm{H}), 4.89(\mathrm{~d}, J=3.8 \mathrm{~Hz}$, $2 \mathrm{H}), 4.81(\mathrm{dd}, J=3.8,2.3 \mathrm{~Hz}, 2 \mathrm{H}), 4.80(\mathrm{dd}, J=3.8,2.3$ $\mathrm{Hz}, 2 \mathrm{H}), 4.76(\mathrm{~s}, 8 \mathrm{H}), 4.75(\mathrm{~d}, J=3.8 \mathrm{~Hz}, 2 \mathrm{H}), 4.74(\mathrm{~d}, J$ $=3.8 \mathrm{~Hz}, 2 \mathrm{H}), 4.67(\mathrm{dd}, J=3.8,2.3 \mathrm{~Hz}, 2 \mathrm{H}), 4.66(\mathrm{dd}, J$ $=3.8,2.3 \mathrm{~Hz}, 2 \mathrm{H}) ;{ }^{13} \mathbf{C}$ NMR (75 $\left.\mathbf{~ M H z}, \mathbf{C D C l}_{3}\right) \delta 143.7$, $134.1,126.8,125.9,123.5,120.9,120.4,91.9,91.7,91.1$,

\section{Procedure for $M \& M$ followed by the formation of epoxide}

Compound $4 \mathbf{b}$ (70 mg, $0.119 \mathrm{mmol})$ was dissolved in freshly distilled $\mathrm{CH}_{2} \mathrm{Cl}_{2}(0.002 \mathrm{M})$ and Grubbs $2^{\text {nd }}$ generation catalyst $1,\left(\mathrm{H}_{2} \mathrm{Imes}\right)\left(\mathrm{PCy}_{3}\right) \mathrm{Cl}_{2} \mathrm{Ru}=\mathrm{CHPh}(2 \times 4.67 \mathrm{mg}, 10 \mathrm{~mol} \%)$ was added. After refluxing under nitrogen for 7 hours, the reaction mixture was concentrated under reduced pressure. The residue was purified by flash column chromatography using pentane/ether/dichloromethane (gradient elusion) to yield $\mathbf{5 b} / \mathbf{5} \mathbf{b}^{\prime}$ with diisopropyl 1,2hydrazinedicarboxylate as a mixture followed by $\mathbf{6 b}$ (light green solid, $18 \mathrm{mg}, 27 \%$ ). The fractions containing the mixture of $\mathbf{5} \mathbf{b} / \mathbf{5} \mathbf{b}^{\prime}$ and diisopropyl 1,2-hydrazinedicarboxylate was concentrated and subsequently treated with $N$-bromosuccinimide $(\sim 1.8$ equiv: determined by the crude NMR of the mixture) and pyridine (2.0 equiv.) in $\mathrm{CH}_{2} \mathrm{Cl}_{2}$. (Note - This operation should be rapidly done due to the instability of $\mathbf{5 b}$ ). The reaction was stopped when diisopropyl 1,2hydrazinedicarboxylate was full converted to DIAD of lower polarity. Aqueous $\mathrm{NaHCO}_{3}$ was added to the reaction mixture. Then, the solution was extracted with $\mathrm{CH}_{2} \mathrm{Cl}_{2}$ twice, dried over $\mathrm{MgSO}_{4}$, and concentrated in vacuo. The residue was purified by flash column chromatography to yield the epoxide 7 (thick red oil, $24 \mathrm{mg}, 34 \%$ ).

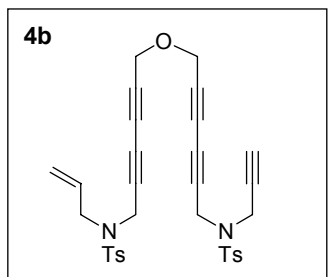

${ }^{1}$ H NMR (300 MHz, CDCl $) \delta 7.70(\mathrm{~d}, J=8.2 \mathrm{~Hz}, 2 \mathrm{H}), 7.68(\mathrm{~d}, J=8.2$ $\mathrm{Hz}, 2 \mathrm{H}), 7.31(\mathrm{~d}, J=8.2 \mathrm{~Hz}, 2 \mathrm{H}), 7.30$ (d, $J=8.2 \mathrm{~Hz}, 2 \mathrm{H}), 5.69$ (ddt, $J=$ $17.1,10.0,4.4 \mathrm{~Hz}, 1 \mathrm{H}), 5.26(\mathrm{~d}, J=17.1 \mathrm{~Hz}, 1 \mathrm{H}), 5.24(\mathrm{~d}, J=10.0 \mathrm{~Hz}$, $1 \mathrm{H}), 4.23$ (brs, 6H), 4.14 (s, 2H), 4.12 (brs, 2H), 3.77 (d, $J=4.4 \mathrm{~Hz}, 2 \mathrm{H}$ ), 2.41 (brs, $2 \times 3 \mathrm{H}) ;{ }^{13} \mathbf{C}$ NMR (75 MHz, $\left.\mathbf{C D C l}_{3}\right) \delta 144.4,144.0,135.5$, $134.8,131.7,129.8,129.7,127.9,127.8,120.5,76.0,74.7,73.7,73.4,72.9$, 72.5, 71.0, 69.6, 69.4, 57.1, 49.6, 37.0, 36.7, 36.6, 21.7; HRMS (ESI) calcd for $\mathrm{C}_{32} \mathrm{H}_{30} \mathrm{~N}_{2} \mathrm{O}_{5} \mathrm{~S}_{2}$ $[\mathrm{M}+\mathrm{H}]^{\bullet+} 587.1641$, found 587.1657.

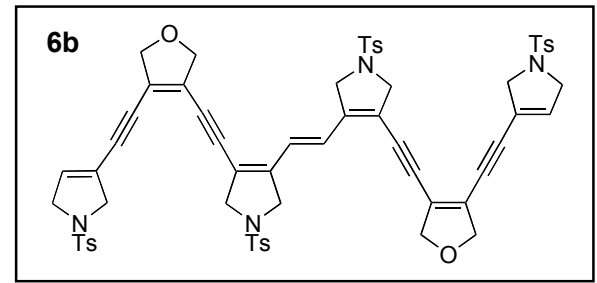

${ }^{1}$ H NMR (300 MHz, CDCl $) \delta 7.73(\mathrm{~d}, J=8.3 \mathrm{~Hz}, 4 \mathrm{H})$, $7.72(\mathrm{~d}, J=8.3 \mathrm{~Hz}, 4 \mathrm{H}), 7.38-7.33(\mathrm{~m}, 8 \mathrm{H}), 6.31(\mathrm{~s}, 2 \mathrm{H})$, 6.02 (brs, 1H), 4.75 (brs, 8H), 4.30 (m, 4H), 4.24 (m, 4H), $4.23(\mathrm{~m}, 4 \mathrm{H}), 4.14(\mathrm{~m}, 4 \mathrm{H}), 2.45(\mathrm{~s}, 6 \mathrm{H}), 2.43(\mathrm{~m}, 6 \mathrm{H}) ;{ }^{13} \mathrm{C}$ NMR (75 MHz, $\left.\mathbf{C D C l}_{3}\right) \delta 144.4,144.1,141.0,133.9$, $133.6,133.1,130.3,130.2,127.7,127.4,124.4,119.6$, $119.0,92.6,91.5,91.1,84.5,57.1,56.5,55.5,55.4,54.1,54.0,21.8$. 


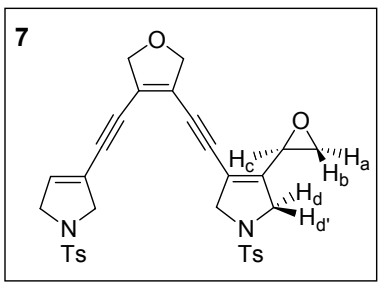

${ }^{1}$ H NMR (300 MHz, CDCl $) \delta 7.75(\mathrm{~d}, J=8.3 \mathrm{~Hz}, 2 \mathrm{H}), 7.73(\mathrm{~d}, J=$ $8.3 \mathrm{~Hz}, 2 \mathrm{H}), 7.36(\mathrm{~d}, J=8.3 \mathrm{~Hz}, 2 \mathrm{H}), 7.34(\mathrm{~d}, J=8.3 \mathrm{~Hz}, 2 \mathrm{H}), 6.03-$ $6.00(\mathrm{~m}, 1 \mathrm{H}), 5.06(\mathrm{dd}, J=11.8,4.9 \mathrm{~Hz}, 1 \mathrm{H}), 4.71(\mathrm{~m}, 4 \mathrm{H}), 4.49-4.39$ $(\mathrm{m}, 1 \mathrm{H}), 4.37-4.27(\mathrm{~m}, 1 \mathrm{H}), 4.26-4.21(\mathrm{~m}, 2 \mathrm{H}), 4.19-4.12(\mathrm{~m}, 4 \mathrm{H})$, $3.75(\mathrm{dd}, J=10.3,4.9 \mathrm{~Hz}, 1 \mathrm{H}), 3.57(\mathrm{dd}, J=11.8,4.9 \mathrm{~Hz}, 1 \mathrm{H}), 2.45$ (s, $2 \times 3 \mathrm{H}) ;{ }^{13} \mathbf{C}$ NMR (75 MHz, $\left.\mathbf{C D C l}_{3}\right) \delta 144.4,144.1,141.5,134.1$, $133.8,132.8,130.3,130.2,127.9,127.3,126.0,92.4,90.8,89.4,84.5$, 56.8, 56.4, 55.5, 53.1, 42.6, 42.5, 31.7, 21.8 ; MS (FAB, $m / z)$ calcd for $\mathrm{C}_{32} \mathrm{H}_{30} \mathrm{~N}_{2} \mathrm{O}_{6} \mathrm{~S}_{2}[\mathrm{M}+2 \mathrm{Na}-$ $\mathrm{H}]^{+} 647$, found 647 .

** Note : $2 \mathrm{D}-\mathrm{COSY}$ data for epoxide shows the correlation between $\mathrm{H}_{\mathrm{c}}$ and $\mathrm{H}_{\mathrm{a}}, \mathrm{H}_{\mathrm{b}}$, as well as between $\mathrm{H}_{\mathrm{c}}$ and $\mathrm{H}_{\mathrm{a}}, \mathrm{H}_{\mathrm{b}}$

\section{$\underline{\text { X-ray diffraction data for } \mathbf{3 c}}$}

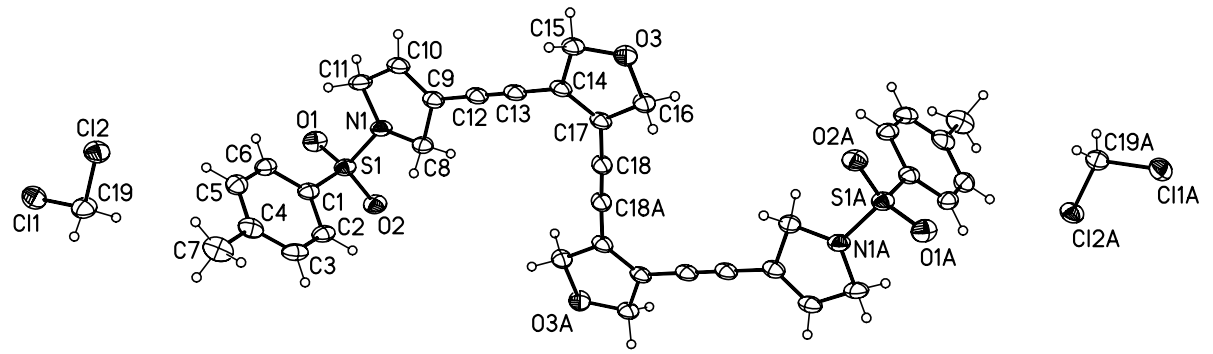

Table 1. Crystal data and structure refinement $3 \mathbf{c}$.

Empirical formula

Formula weight

Temperature

Wavelength

Crystal system

Space group

Unit cell dimensions

Volume

Z

Density (calculated)
$\mathrm{C}_{36} \mathrm{H}_{32} \mathrm{~N}_{2} \mathrm{O}_{6} \mathrm{~S}_{2} .2 \mathrm{CH}_{2} \mathrm{Cl}_{2}$

822.61

$100(2) \mathrm{K}$

$0.71073 \AA$

Triclinic

$\mathrm{P} \overline{1}$

$\mathrm{a}=6.2717(11) \AA \quad \alpha=95.316(2)^{\circ}$.

$\mathrm{b}=9.5873(17) \AA \quad \beta=97.598(2)^{\circ}$.

$\mathrm{c}=16.319(3) \AA \quad \gamma=96.233(2)^{\circ}$.

$961.2(3) \AA^{3}$

1

$1.421 \mathrm{Mg} / \mathrm{m}^{3}$ 


$\begin{array}{ll}\text { Absorption coefficient } & 0.465 \mathrm{~mm}^{-1} \\ \mathrm{~F}(000) & 426 \\ \text { Crystal size } & 0.46 \times 0.28 \times 0.25 \mathrm{~mm}^{3} \\ \text { Theta range for data collection } & 1.27 \text { to } 26.39^{\circ} . \\ \text { Index ranges } & -7<=\mathrm{h}<=7,-11<=\mathrm{k}<=11,-20<=1<=20 \\ \text { Reflections collected } & 7934 \\ \text { Independent reflections } & 3881[\mathrm{R}(\mathrm{int})=0.0160] \\ \text { Completeness to theta }=26.39^{\circ} & 98.7 \% \\ \text { Absorption correction } & \text { Multi-scan with SADABS } \\ \text { Max. and min. transmission } & 0.8926 \text { and } 0.8145 \\ \text { Refinement method } & \text { Full-matrix least-squares on } \mathrm{F}^{2} \\ \text { Data / restraints / parameters } & 3881 / 0 / 236 \\ \text { Goodness-of-fit on F } 2 & 1.078 \\ \text { Final R indices [I }>2 \text { sigma(I)] } & \mathrm{R} 1=0.0370, \mathrm{wR} 2=0.1000 \\ \text { R indices (all data) } & \mathrm{R} 1=0.0429, \mathrm{wR} 2=0.1064 \\ \text { Largest diff. peak and hole } & 0.729 \text { and }-0.248 \mathrm{e} . \AA^{-3}\end{array}$

Table 2. Atomic coordinates $\left(\mathrm{x} 10^{4}\right)$ and equivalent isotropic displacement parameters $\left(\AA^{2} \times 10^{3}\right)$ for 3c. $U(\mathrm{eq})$ is defined as one third of the trace of the orthogonalized $\mathrm{U}^{\mathrm{ij}}$ tensor.

\begin{tabular}{|c|c|c|c|c|}
\hline & $\mathrm{x}$ & $\mathrm{y}$ & $\mathrm{z}$ & $\mathrm{U}(\mathrm{eq})$ \\
\hline $\mathrm{S}(1)$ & $4513(1)$ & 10092(1) & $6916(1)$ & $29(1)$ \\
\hline $\mathrm{Cl}(1)$ & $-869(1)$ & 5576(1) & $2155(1)$ & $40(1)$ \\
\hline $\mathrm{Cl}(2)$ & $1587(1)$ & $4027(1)$ & $3338(1)$ & $39(1)$ \\
\hline $\mathrm{O}(1)$ & $2202(2)$ & $10046(1)$ & $6798(1)$ & $37(1)$ \\
\hline $\mathrm{O}(2)$ & $5868(2)$ & $11420(1)$ & $7055(1)$ & $36(1)$ \\
\hline $\mathrm{O}(3)$ & $14693(2)$ & $6035(1)$ & $11010(1)$ & $38(1)$ \\
\hline $\mathrm{N}(1)$ & $5130(2)$ & $9279(2)$ & 7731(1) & $30(1)$ \\
\hline$C(1)$ & $5248(3)$ & $9087(2)$ & $6063(1)$ & $27(1)$ \\
\hline$C(2)$ & $7305(3)$ & $9389(2)$ & $5841(1)$ & $31(1)$ \\
\hline$C(3)$ & $7911(3)$ & $8534(2)$ & $5206(1)$ & $34(1)$ \\
\hline$C(4)$ & $6538(3)$ & $7380(2)$ & $4788(1)$ & $34(1)$ \\
\hline$C(5)$ & $4485(3)$ & $7099(2)$ & $5018(1)$ & $32(1)$ \\
\hline$C(6)$ & $3833(3)$ & $7946(2)$ & $5652(1)$ & $30(1)$ \\
\hline$C(7)$ & $7252(4)$ & $6462(2)$ & $4102(2)$ & $46(1)$ \\
\hline$C(8)$ & $7433(3)$ & $9305(2)$ & $8085(1)$ & $30(1)$ \\
\hline C(9) & $7339(3)$ & $8004(2)$ & $8546(1)$ & $29(1)$ \\
\hline$C(10)$ & $5386(3)$ & $7248(2)$ & $8377(1)$ & $32(1)$ \\
\hline $\mathrm{C}(11)$ & $3868(3)$ & $7902(2)$ & $7797(1)$ & $35(1)$ \\
\hline$C(12)$ & $9157(3)$ & $7699(2)$ & $9087(1)$ & $30(1)$ \\
\hline$C(13)$ & $10692(3)$ & $7464(2)$ & 9550(1) & $30(1)$ \\
\hline$C(14)$ & 12491(3) & $7192(2)$ & $10102(1)$ & $28(1)$ \\
\hline$C(15)$ & $12679(3)$ & $5823(2)$ & $10467(1)$ & $32(1)$ \\
\hline$C(16)$ & $15834(3)$ & $7401(2)$ & $10958(1)$ & $33(1)$ \\
\hline$C(17)$ & $14322(3)$ & $8081(2)$ & $10372(1)$ & $29(1)$ \\
\hline
\end{tabular}




$\begin{array}{lrrrr}\mathrm{C}(18) & 14801(3) & 9439(2) & 10122(1) & 31(1) \\ \mathrm{C}(19) & 1696(3) & 5447(2) & 2718(1) & 40(1)\end{array}$

Table 3. Bond lengths $[\AA]$ and angles $\left[{ }^{\circ}\right]$ for 3c.

$\begin{array}{ll}\mathrm{S}(1)-\mathrm{O}(1) & 1.4317(14) \\ \mathrm{S}(1)-\mathrm{O}(2) & 1.4339(14) \\ \mathrm{S}(1)-\mathrm{N}(1) & 1.6261(16) \\ \mathrm{S}(1)-\mathrm{C}(1) & 1.7603(19) \\ \mathrm{Cl}(1)-\mathrm{C}(19) & 1.766(2) \\ \mathrm{Cl}(2)-\mathrm{C}(19) & 1.771(2) \\ \mathrm{O}(3)-\mathrm{C}(15) & 1.427(2) \\ \mathrm{O}(3)-\mathrm{C}(16) & 1.438(2) \\ \mathrm{N}(1)-\mathrm{C}(8) & 1.480(2) \\ \mathrm{N}(1)-\mathrm{C}(11) & 1.484(2) \\ \mathrm{C}(1)-\mathrm{C}(6) & 1.389(3) \\ \mathrm{C}(1)-\mathrm{C}(2) & 1.394(2) \\ \mathrm{C}(2)-\mathrm{C}(3) & 1.380(3) \\ \mathrm{C}(2)-\mathrm{H}(2) & 0.9500 \\ \mathrm{C}(3)-\mathrm{C}(4) & 1.389(3) \\ \mathrm{C}(3)-\mathrm{H}(3) & 0.9500 \\ \mathrm{C}(4)-\mathrm{C}(5) & 1.393(3) \\ \mathrm{C}(4)-\mathrm{C}(7) & 1.505(3) \\ \mathrm{C}(5)-\mathrm{C}(6) & 1.388(3) \\ \mathrm{C}(5)-\mathrm{H}(5) & 0.9500 \\ \mathrm{C}(6)-\mathrm{H}(6) & 0.9500 \\ \mathrm{C}(7)-\mathrm{H}(7 \mathrm{~A}) & 0.9800 \\ \mathrm{C}(7)-\mathrm{H}(7 \mathrm{~B}) & 0.9800 \\ \mathrm{C}(7)-\mathrm{H}(7 \mathrm{C}) & 0.9800 \\ \mathrm{C}(8)-\mathrm{C}(9) & 1.515(2) \\ \mathrm{C}(8)-\mathrm{H}(8 \mathrm{~A}) & 0.9900 \\ \mathrm{C}(8)-\mathrm{H}(8 \mathrm{~B}) & 0.9900 \\ \mathrm{C}(9)-\mathrm{C}(10) & 1.335(3) \\ \mathrm{C}(9)-\mathrm{C}(12) & 1.421(3) \\ \mathrm{C}(10)-\mathrm{C}(11) & 1.482(3) \\ \mathrm{C}(10)-\mathrm{H}(10) & 0.9500 \\ \mathrm{C}(11)-\mathrm{H}(11 \mathrm{~A}) & 0.9900 \\ \mathrm{C}(11)-\mathrm{H}(11 \mathrm{~B}) & 0.9900 \\ \mathrm{C}(12)-\mathrm{C}(13) & 1.198(3) \\ \mathrm{C}(13)-\mathrm{C}(14) & 1.411(3) \\ \mathrm{C}(14)-\mathrm{C}(17) & 1.350(3) \\ \mathrm{C}(14)-\mathrm{C}(15) & 1.501(2) \\ \mathrm{C}(15)-\mathrm{H}(15 \mathrm{~A}) & 0.9900 \\ \mathrm{C}(15)-\mathrm{H}(15 \mathrm{~B}) & 0.9900 \\ \mathrm{C}(16)-\mathrm{C}(17) & 1.499(3) \\ \mathrm{C}(16)-\mathrm{H}(16 \mathrm{~A}) & 0.9900 \\ \mathrm{C}(16)-\mathrm{H}(16 \mathrm{~B}) & 0.9900 \\ \mathrm{C}(17)-\mathrm{C}(18) & \\ & \end{array}$




\begin{tabular}{|c|c|}
\hline $\mathrm{C}(18)-\mathrm{C}(18) \# 1$ & $1.193(4)$ \\
\hline $\mathrm{C}(19)-\mathrm{H}(19 \mathrm{~A})$ & 0.9900 \\
\hline C(19)-H(19B) & 0.9900 \\
\hline $\mathrm{O}(1)-\mathrm{S}(1)-\mathrm{O}(2)$ & $120.37(8)$ \\
\hline $\mathrm{O}(1)-\mathrm{S}(1)-\mathrm{N}(1)$ & $106.17(8)$ \\
\hline $\mathrm{O}(2)-\mathrm{S}(1)-\mathrm{N}(1)$ & $106.07(8)$ \\
\hline $\mathrm{O}(1)-\mathrm{S}(1)-\mathrm{C}(1)$ & $108.12(9)$ \\
\hline $\mathrm{O}(2)-\mathrm{S}(1)-\mathrm{C}(1)$ & $108.45(8)$ \\
\hline $\mathrm{N}(1)-\mathrm{S}(1)-\mathrm{C}(1)$ & $106.92(8)$ \\
\hline $\mathrm{C}(15)-\mathrm{O}(3)-\mathrm{C}(16)$ & $110.63(13)$ \\
\hline $\mathrm{C}(8)-\mathrm{N}(1)-\mathrm{C}(11)$ & $111.24(14)$ \\
\hline $\mathrm{C}(8)-\mathrm{N}(1)-\mathrm{S}(1)$ & $119.46(12)$ \\
\hline $\mathrm{C}(11)-\mathrm{N}(1)-\mathrm{S}(1)$ & $118.71(13)$ \\
\hline $\mathrm{C}(6)-\mathrm{C}(1)-\mathrm{C}(2)$ & $120.56(17)$ \\
\hline$C(6)-C(1)-S(1)$ & $119.88(13)$ \\
\hline $\mathrm{C}(2)-\mathrm{C}(1)-\mathrm{S}(1)$ & $119.43(14)$ \\
\hline $\mathrm{C}(3)-\mathrm{C}(2)-\mathrm{C}(1)$ & $118.80(18)$ \\
\hline $\mathrm{C}(3)-\mathrm{C}(2)-\mathrm{H}(2)$ & 120.6 \\
\hline $\mathrm{C}(1)-\mathrm{C}(2)-\mathrm{H}(2)$ & 120.6 \\
\hline$C(2)-C(3)-C(4)$ & $121.90(17)$ \\
\hline $\mathrm{C}(2)-\mathrm{C}(3)-\mathrm{H}(3)$ & 119.1 \\
\hline $\mathrm{C}(4)-\mathrm{C}(3)-\mathrm{H}(3)$ & 119.1 \\
\hline $\mathrm{C}(3)-\mathrm{C}(4)-\mathrm{C}(5)$ & $118.40(18)$ \\
\hline $\mathrm{C}(3)-\mathrm{C}(4)-\mathrm{C}(7)$ & $120.63(18)$ \\
\hline$C(5)-C(4)-C(7)$ & $120.96(19)$ \\
\hline$C(6)-C(5)-C(4)$ & $120.82(18)$ \\
\hline $\mathrm{C}(6)-\mathrm{C}(5)-\mathrm{H}(5)$ & 119.6 \\
\hline $\mathrm{C}(4)-\mathrm{C}(5)-\mathrm{H}(5)$ & 119.6 \\
\hline$C(5)-C(6)-C(1)$ & $119.51(17)$ \\
\hline $\mathrm{C}(5)-\mathrm{C}(6)-\mathrm{H}(6)$ & 120.2 \\
\hline $\mathrm{C}(1)-\mathrm{C}(6)-\mathrm{H}(6)$ & 120.2 \\
\hline $\mathrm{C}(4)-\mathrm{C}(7)-\mathrm{H}(7 \mathrm{~A})$ & 109.5 \\
\hline $\mathrm{C}(4)-\mathrm{C}(7)-\mathrm{H}(7 \mathrm{~B})$ & 109.5 \\
\hline $\mathrm{H}(7 \mathrm{~A})-\mathrm{C}(7)-\mathrm{H}(7 \mathrm{~B})$ & 109.5 \\
\hline $\mathrm{C}(4)-\mathrm{C}(7)-\mathrm{H}(7 \mathrm{C})$ & 109.5 \\
\hline $\mathrm{H}(7 \mathrm{~A})-\mathrm{C}(7)-\mathrm{H}(7 \mathrm{C})$ & 109.5 \\
\hline $\mathrm{H}(7 \mathrm{~B})-\mathrm{C}(7)-\mathrm{H}(7 \mathrm{C})$ & 109.5 \\
\hline $\mathrm{N}(1)-\mathrm{C}(8)-\mathrm{C}(9)$ & $101.29(14)$ \\
\hline $\mathrm{N}(1)-\mathrm{C}(8)-\mathrm{H}(8 \mathrm{~A})$ & 111.5 \\
\hline $\mathrm{C}(9)-\mathrm{C}(8)-\mathrm{H}(8 \mathrm{~A})$ & 111.5 \\
\hline $\mathrm{N}(1)-\mathrm{C}(8)-\mathrm{H}(8 \mathrm{~B})$ & 111.5 \\
\hline $\mathrm{C}(9)-\mathrm{C}(8)-\mathrm{H}(8 \mathrm{~B})$ & 111.5 \\
\hline $\mathrm{H}(8 \mathrm{~A})-\mathrm{C}(8)-\mathrm{H}(8 \mathrm{~B})$ & 109.3 \\
\hline $\mathrm{C}(10)-\mathrm{C}(9)-\mathrm{C}(12)$ & $126.99(16)$ \\
\hline $\mathrm{C}(10)-\mathrm{C}(9)-\mathrm{C}(8)$ & $111.36(16)$ \\
\hline $\mathrm{C}(12)-\mathrm{C}(9)-\mathrm{C}(8)$ & $121.64(16)$ \\
\hline $\mathrm{C}(9)-\mathrm{C}(10)-\mathrm{C}(11)$ & $112.12(16)$ \\
\hline $\mathrm{C}(9)-\mathrm{C}(10)-\mathrm{H}(10)$ & 123.9 \\
\hline $\mathrm{C}(11)-\mathrm{C}(10)-\mathrm{H}(10)$ & 123.9 \\
\hline $\mathrm{C}(10)-\mathrm{C}(11)-\mathrm{N}(1)$ & $102.27(15)$ \\
\hline $\mathrm{C}(10)-\mathrm{C}(11)-\mathrm{H}(11 \mathrm{~A})$ & 111.3 \\
\hline $\mathrm{N}(1)-\mathrm{C}(11)-\mathrm{H}(11 \mathrm{~A})$ & 111.3 \\
\hline
\end{tabular}




$\begin{array}{ll}\mathrm{C}(10)-\mathrm{C}(11)-\mathrm{H}(11 \mathrm{~B}) & 111.3 \\ \mathrm{~N}(1)-\mathrm{C}(11)-\mathrm{H}(11 \mathrm{~B}) & 111.3 \\ \mathrm{H}(11 \mathrm{~A})-\mathrm{C}(11)-\mathrm{H}(11 \mathrm{~B}) & 109.2 \\ \mathrm{C}(13)-\mathrm{C}(12)-\mathrm{C}(9) & 178.9(2) \\ \mathrm{C}(12)-\mathrm{C}(13)-\mathrm{C}(14) & 179.4(2) \\ \mathrm{C}(17)-\mathrm{C}(14)-\mathrm{C}(13) & 126.56(16) \\ \mathrm{C}(17)-\mathrm{C}(14)-\mathrm{C}(15) & 108.99(16) \\ \mathrm{C}(13)-\mathrm{C}(14)-\mathrm{C}(15) & 124.43(16) \\ \mathrm{O}(3)-\mathrm{C}(15)-\mathrm{C}(14) & 105.45(14) \\ \mathrm{O}(3)-\mathrm{C}(15)-\mathrm{H}(15 \mathrm{~A}) & 110.7 \\ \mathrm{C}(14)-\mathrm{C}(15)-\mathrm{H}(15 \mathrm{~A}) & 110.7 \\ \mathrm{O}(3)-\mathrm{C}(15)-\mathrm{H}(15 \mathrm{~B}) & 110.7 \\ \mathrm{C}(14)-\mathrm{C}(15)-\mathrm{H}(15 \mathrm{~B}) & 110.7 \\ \mathrm{H}(15 \mathrm{~A})-\mathrm{C}(15)-\mathrm{H}(15 \mathrm{~B}) & 108.8 \\ \mathrm{O}(3)-\mathrm{C}(16)-\mathrm{C}(17) & 104.58(15) \\ \mathrm{O}(3)-\mathrm{C}(16)-\mathrm{H}(16 \mathrm{~A}) & 110.8 \\ \mathrm{C}(17)-\mathrm{C}(16)-\mathrm{H}(16 \mathrm{~A}) & 110.8 \\ \mathrm{O}(3)-\mathrm{C}(16)-\mathrm{H}(16 \mathrm{~B}) & 110.8 \\ \mathrm{C}(17)-\mathrm{C}(16)-\mathrm{H}(16 \mathrm{~B}) & 110.8 \\ \mathrm{H}(16 \mathrm{~A})-\mathrm{C}(16)-\mathrm{H}(16 \mathrm{~B}) & 108.9 \\ \mathrm{C}(14)-\mathrm{C}(17)-\mathrm{C}(18) & 125.52(18) \\ \mathrm{C}(14)-\mathrm{C}(17)-\mathrm{C}(16) & 110.18(15) \\ \mathrm{C}(18)-\mathrm{C}(17)-\mathrm{C}(16) & 124.27(17) \\ \mathrm{C}(18) \# 1-\mathrm{C}(18)-\mathrm{C}(17) & 177.3(3) \\ \mathrm{Cl}(1)-\mathrm{C}(19)-\mathrm{Cl}(2) & 111.48(11) \\ \mathrm{Cl}(1)-\mathrm{C}(19)-\mathrm{H}(19 \mathrm{~A}) & 109.3 \\ \mathrm{Cl}(2)-\mathrm{C}(19)-\mathrm{H}(19 \mathrm{~A}) & 109.3 \\ \mathrm{Cl}(1)-\mathrm{C}(19)-\mathrm{H}(19 \mathrm{~B}) & 109.3 \\ \mathrm{Cl}(2)-\mathrm{C}(19)-\mathrm{H}(19 \mathrm{~B}) & 109.3 \\ \mathrm{H}(19 \mathrm{~A})-\mathrm{C}(19)-\mathrm{H}(19 \mathrm{~B}) & 108.0 \\ & \end{array}$

Symmetry transformations used to generate equivalent atoms:

\#1 - $\mathrm{x}+3,-\mathrm{y}+2,-\mathrm{z}+2$

Table 4. Anisotropic displacement parameters $\left(\AA^{2} \times 10^{3}\right)$ for 3c. The anisotropic displacement factor exponent takes the form: $-2 \pi^{2}\left[h^{2} a^{* 2} U^{11}+\ldots+2 h k a^{*} b^{*} U^{12}\right]$

\begin{tabular}{lcccccc}
\hline & $\mathrm{U}^{11}$ & $\mathrm{U}^{22}$ & $\mathrm{U}^{33}$ & $\mathrm{U}^{23}$ & $\mathrm{U}^{13}$ & $\mathrm{U}^{12}$ \\
\hline $\mathrm{S}(1)$ & $30(1)$ & $24(1)$ & $36(1)$ & $11(1)$ & $7(1)$ & $4(1)$ \\
$\mathrm{Cl}(1)$ & $45(1)$ & $41(1)$ & $38(1)$ & $11(1)$ & $5(1)$ & $9(1)$ \\
$\mathrm{Cl}(2)$ & $48(1)$ & $30(1)$ & $41(1)$ & $14(1)$ & $7(1)$ & $5(1)$ \\
$\mathrm{O}(1)$ & $32(1)$ & $36(1)$ & $47(1)$ & $11(1)$ & $10(1)$ & $9(1)$ \\
$\mathrm{O}(2)$ & $43(1)$ & $23(1)$ & $45(1)$ & $12(1)$ & $6(1)$ & $2(1)$ \\
$\mathrm{O}(3)$ & $50(1)$ & $22(1)$ & $41(1)$ & $16(1)$ & $-4(1)$ & $-5(1)$ \\
$\mathrm{N}(1)$ & $31(1)$ & $27(1)$ & $35(1)$ & $13(1)$ & $8(1)$ & $0(1)$ \\
$\mathrm{C}(1)$ & $27(1)$ & $25(1)$ & $33(1)$ & $14(1)$ & $5(1)$ & $5(1)$ \\
$\mathrm{C}(2)$ & $27(1)$ & $27(1)$ & $41(1)$ & $18(1)$ & $6(1)$ & $3(1)$ \\
$\mathrm{C}(3)$ & $29(1)$ & $36(1)$ & $45(1)$ & $23(1)$ & $13(1)$ & $9(1)$
\end{tabular}




\begin{tabular}{lcccccc}
$\mathrm{C}(4)$ & $38(1)$ & $33(1)$ & $37(1)$ & $17(1)$ & $9(1)$ & $14(1)$ \\
$\mathrm{C}(5)$ & $32(1)$ & $30(1)$ & $35(1)$ & $11(1)$ & $2(1)$ & $4(1)$ \\
$\mathrm{C}(6)$ & $25(1)$ & $30(1)$ & $36(1)$ & $13(1)$ & $5(1)$ & $2(1)$ \\
$\mathrm{C}(7)$ & $55(1)$ & $44(1)$ & $49(1)$ & $14(1)$ & $19(1)$ & $20(1)$ \\
$\mathrm{C}(8)$ & $34(1)$ & $23(1)$ & $33(1)$ & $12(1)$ & $4(1)$ & $0(1)$ \\
$\mathrm{C}(9)$ & $40(1)$ & $21(1)$ & $28(1)$ & $7(1)$ & $9(1)$ & $2(1)$ \\
$\mathrm{C}(10)$ & $43(1)$ & $24(1)$ & $30(1)$ & $9(1)$ & $12(1)$ & $-1(1)$ \\
$\mathrm{C}(11)$ & $36(1)$ & $31(1)$ & $41(1)$ & $15(1)$ & $11(1)$ & $-4(1)$ \\
$\mathrm{C}(12)$ & $43(1)$ & $19(1)$ & $29(1)$ & $8(1)$ & $9(1)$ & $-2(1)$ \\
$\mathrm{C}(13)$ & $44(1)$ & $18(1)$ & $28(1)$ & $8(1)$ & $10(1)$ & $-2(1)$ \\
$\mathrm{C}(14)$ & $43(1)$ & $18(1)$ & $24(1)$ & $7(1)$ & $8(1)$ & $-1(1)$ \\
$\mathrm{C}(15)$ & $43(1)$ & $20(1)$ & $32(1)$ & $11(1)$ & $6(1)$ & $-2(1)$ \\
$\mathrm{C}(16)$ & $46(1)$ & $19(1)$ & $31(1)$ & $8(1)$ & $1(1)$ & $-3(1)$ \\
$\mathrm{C}(17)$ & $46(1)$ & $18(1)$ & $24(1)$ & $6(1)$ & $7(1)$ & $-2(1)$ \\
$\mathrm{C}(18)$ & $44(1)$ & $20(1)$ & $26(1)$ & $4(1)$ & $2(1)$ & $-3(1)$ \\
$\mathrm{C}(19)$ & $43(1)$ & $31(1)$ & $46(1)$ & $17(1)$ & $3(1)$ & $1(1)$ \\
& & & & & & \\
\hline
\end{tabular}

Table 5. Hydrogen coordinates $\left(\mathrm{x} 10^{4}\right)$ and isotropic displacement parameters $\left(\AA^{2} \mathrm{x} 10^{3}\right)$ for $3 c$.

\begin{tabular}{|c|c|c|c|c|}
\hline & $\mathrm{x}$ & $\mathrm{y}$ & $\mathrm{z}$ & $\mathrm{U}(\mathrm{eq})$ \\
\hline $\mathrm{H}(2)$ & 8273 & 10170 & 6121 & 37 \\
\hline $\mathrm{H}(3)$ & 9309 & 8741 & 5050 & 41 \\
\hline $\mathrm{H}(5)$ & 3519 & 6318 & 4736 & 39 \\
\hline $\mathrm{H}(6)$ & 2429 & 7747 & 5804 & 36 \\
\hline $\mathrm{H}(7 \mathrm{~A})$ & 6355 & 5543 & 4016 & 69 \\
\hline $\mathrm{H}(7 \mathrm{~B})$ & 8774 & 6328 & 4256 & 69 \\
\hline $\mathrm{H}(7 \mathrm{C})$ & 7091 & 6917 & 3588 & 69 \\
\hline $\mathrm{H}(8 \mathrm{~A})$ & 7959 & 10173 & 8468 & 36 \\
\hline $\mathrm{H}(8 \mathrm{~B})$ & 8369 & 9230 & 7643 & 36 \\
\hline $\mathrm{H}(10)$ & 5013 & 6390 & 8602 & 38 \\
\hline $\mathrm{H}(11 \mathrm{~A})$ & 2512 & 8034 & 8028 & 42 \\
\hline $\mathrm{H}(11 \mathrm{~B})$ & 3510 & 7328 & 7250 & 42 \\
\hline $\mathrm{H}(15 \mathrm{~A})$ & 11460 & 5593 & 10780 & 38 \\
\hline $\mathrm{H}(15 \mathrm{~B})$ & 12673 & 5043 & 10024 & 38 \\
\hline $\mathrm{H}(16 \mathrm{~A})$ & 17216 & 7310 & 10738 & 39 \\
\hline $\mathrm{H}(16 \mathrm{~B})$ & 16147 & 7959 & 11510 & 39 \\
\hline $\mathrm{H}(19 \mathrm{~A})$ & 2210 & 6340 & 3079 & 48 \\
\hline$H(19 B)$ & 2746 & 5308 & 2324 & 48 \\
\hline
\end{tabular}


Table 6. Torsion angles $\left[{ }^{\circ}\right]$ for $\mathbf{3 c}$.

\begin{tabular}{|c|c|}
\hline $\mathrm{O}(1)-\mathrm{S}(1)-\mathrm{N}(1)-\mathrm{C}(8)$ & $-172.01(13)$ \\
\hline $\mathrm{O}(2)-\mathrm{S}(1)-\mathrm{N}(1)-\mathrm{C}(8)$ & $-42.86(16)$ \\
\hline $\mathrm{C}(1)-\mathrm{S}(1)-\mathrm{N}(1)-\mathrm{C}(8)$ & $72.73(15)$ \\
\hline $\mathrm{O}(1)-\mathrm{S}(1)-\mathrm{N}(1)-\mathrm{C}(11)$ & $46.38(16)$ \\
\hline $\mathrm{O}(2)-\mathrm{S}(1)-\mathrm{N}(1)-\mathrm{C}(11)$ & $175.53(13)$ \\
\hline $\mathrm{C}(1)-\mathrm{S}(1)-\mathrm{N}(1)-\mathrm{C}(11)$ & $-68.88(15)$ \\
\hline $\mathrm{O}(1)-\mathrm{S}(1)-\mathrm{C}(1)-\mathrm{C}(6)$ & $-29.83(16)$ \\
\hline $\mathrm{O}(2)-\mathrm{S}(1)-\mathrm{C}(1)-\mathrm{C}(6)$ & $-161.90(14)$ \\
\hline $\mathrm{N}(1)-\mathrm{S}(1)-\mathrm{C}(1)-\mathrm{C}(6)$ & $84.11(15)$ \\
\hline $\mathrm{O}(1)-\mathrm{S}(1)-\mathrm{C}(1)-\mathrm{C}(2)$ & $154.33(14)$ \\
\hline $\mathrm{O}(2)-\mathrm{S}(1)-\mathrm{C}(1)-\mathrm{C}(2)$ & $22.27(16)$ \\
\hline $\mathrm{N}(1)-\mathrm{S}(1)-\mathrm{C}(1)-\mathrm{C}(2)$ & $-91.73(15)$ \\
\hline $\mathrm{C}(6)-\mathrm{C}(1)-\mathrm{C}(2)-\mathrm{C}(3)$ & $-0.1(3)$ \\
\hline $\mathrm{S}(1)-\mathrm{C}(1)-\mathrm{C}(2)-\mathrm{C}(3)$ & $175.74(13)$ \\
\hline $\mathrm{C}(1)-\mathrm{C}(2)-\mathrm{C}(3)-\mathrm{C}(4)$ & $-0.4(3)$ \\
\hline$C(2)-C(3)-C(4)-C(5)$ & $0.7(3)$ \\
\hline $\mathrm{C}(2)-\mathrm{C}(3)-\mathrm{C}(4)-\mathrm{C}(7)$ & $-179.57(17)$ \\
\hline $\mathrm{C}(3)-\mathrm{C}(4)-\mathrm{C}(5)-\mathrm{C}(6)$ & $-0.4(3)$ \\
\hline$C(7)-C(4)-C(5)-C(6)$ & $179.84(17)$ \\
\hline $\mathrm{C}(4)-\mathrm{C}(5)-\mathrm{C}(6)-\mathrm{C}(1)$ & $-0.1(3)$ \\
\hline$C(2)-C(1)-C(6)-C(5)$ & $0.3(3)$ \\
\hline $\mathrm{S}(1)-\mathrm{C}(1)-\mathrm{C}(6)-\mathrm{C}(5)$ & $-175.47(13)$ \\
\hline$C(11)-N(1)-C(8)-C(9)$ & $-12.97(19)$ \\
\hline $\mathrm{S}(1)-\mathrm{N}(1)-\mathrm{C}(8)-\mathrm{C}(9)$ & $-157.21(12)$ \\
\hline $\mathrm{N}(1)-\mathrm{C}(8)-\mathrm{C}(9)-\mathrm{C}(10)$ & $8.3(2)$ \\
\hline $\mathrm{N}(1)-\mathrm{C}(8)-\mathrm{C}(9)-\mathrm{C}(12)$ & $-171.21(16)$ \\
\hline $\mathrm{C}(12)-\mathrm{C}(9)-\mathrm{C}(10)-\mathrm{C}(11)$ & $178.78(18)$ \\
\hline$C(8)-C(9)-C(10)-C(11)$ & $-0.7(2)$ \\
\hline $\mathrm{C}(9)-\mathrm{C}(10)-\mathrm{C}(11)-\mathrm{N}(1)$ & $-7.2(2)$ \\
\hline $\mathrm{C}(8)-\mathrm{N}(1)-\mathrm{C}(11)-\mathrm{C}(10)$ & $12.7(2)$ \\
\hline $\mathrm{S}(1)-\mathrm{N}(1)-\mathrm{C}(11)-\mathrm{C}(10)$ & $157.29(13)$ \\
\hline $\mathrm{C}(10)-\mathrm{C}(9)-\mathrm{C}(12)-\mathrm{C}(13)$ & $-124(10)$ \\
\hline $\mathrm{C}(8)-\mathrm{C}(9)-\mathrm{C}(12)-\mathrm{C}(13)$ & $56(10)$ \\
\hline $\mathrm{C}(9)-\mathrm{C}(12)-\mathrm{C}(13)-\mathrm{C}(14)$ & $46(26)$ \\
\hline$C(12)-C(13)-C(14)-C(17)$ & $-92(20)$ \\
\hline $\mathrm{C}(12)-\mathrm{C}(13)-\mathrm{C}(14)-\mathrm{C}(15)$ & $90(20)$ \\
\hline $\mathrm{C}(16)-\mathrm{O}(3)-\mathrm{C}(15)-\mathrm{C}(14)$ & $-4.3(2)$ \\
\hline $\mathrm{C}(17)-\mathrm{C}(14)-\mathrm{C}(15)-\mathrm{O}(3)$ & $3.9(2)$ \\
\hline $\mathrm{C}(13)-\mathrm{C}(14)-\mathrm{C}(15)-\mathrm{O}(3)$ & $-177.61(17)$ \\
\hline $\mathrm{C}(15)-\mathrm{O}(3)-\mathrm{C}(16)-\mathrm{C}(17)$ & $3.2(2)$ \\
\hline $\mathrm{C}(13)-\mathrm{C}(14)-\mathrm{C}(17)-\mathrm{C}(18)$ & $-2.5(3)$ \\
\hline$C(15)-C(14)-C(17)-C(18)$ & $175.98(18)$ \\
\hline $\mathrm{C}(13)-\mathrm{C}(14)-\mathrm{C}(17)-\mathrm{C}(16)$ & $179.53(18)$ \\
\hline$C(15)-C(14)-C(17)-C(16)$ & $-2.0(2)$ \\
\hline $\mathrm{O}(3)-\mathrm{C}(16)-\mathrm{C}(17)-\mathrm{C}(14)$ & $-0.6(2)$ \\
\hline $\mathrm{O}(3)-\mathrm{C}(16)-\mathrm{C}(17)-\mathrm{C}(18)$ & $-178.64(17)$ \\
\hline $\mathrm{C}(14)-\mathrm{C}(17)-\mathrm{C}(18)-\mathrm{C}(18) \# 1$ & $-59(6)$ \\
\hline $\mathrm{C}(16)-\mathrm{C}(17)-\mathrm{C}(18)-\mathrm{C}(18) \# 1$ & $118(6)$ \\
\hline
\end{tabular}

Symmetry transformations used to generate equivalent atoms: $\# 1-\mathrm{x}+3,-\mathrm{y}+2,-\mathrm{z}+2$ 